20 Juin 2018

\title{
Exponents of Diophantine Approximation and Sturmian Continued Fractions
}

\author{
Yann Bugeaud \& Michel Laurent
}

\begin{abstract}
Let $\xi$ be a real number and let $n$ be a positive integer. We define four exponents of Diophantine approximation, which complement the exponents $w_{n}(\xi)$ and $w_{n}^{*}(\xi)$ defined by Mahler and Koksma. We calculate their six values when $n=2$ and $\xi$ is a real number whose continued fraction expansion coincides with some Sturmian sequence of positive integers, up to the initial terms. In particular, we obtain the exact exponent of approximation to such a continued fraction $\xi$ by quadratic surds.
\end{abstract}

\section{Introduction}

Mahler [19] and Koksma [16] have introduced two classifications of real numbers $\xi$ in terms of their properties of approximation by algebraic numbers. Keeping their notations, for every integer $n \geq 1$, let us denote by $w_{n}(\xi)$ the supremum of the real numbers $w$ for which the inequality

$$
0<|P(\xi)| \leq H(P)^{-w}
$$

is satisfied for infinitely many polynomials $P(X)$ with integer coefficients and degree at most $n$ (the height $H(P)$ of a polynomial $P(X)$ is the maximum of the moduli of its coefficients). In a similar way, define $w_{n}^{*}(\xi)$ as the supremum of the real numbers $w^{*}$ for which the inequality

$$
0<|\xi-\alpha| \leq H(\alpha)^{-w^{*}-1}
$$

is satisfied for infinitely many algebraic numbers $\alpha$ of degree at most $n$ (the height $H(\alpha)$ of an algebraic number $\alpha$ is the height of its minimal polynomial over $\mathbf{Z}$ ). The adjunction of -1 in the exponent of the right hand side of (1) has the following motivation. Assume that $|P(\xi)|$ is small. Then, the polynomial $P(X)$ has some root $\alpha$ with $|\xi-\alpha| \ll\left|P(\xi) / P^{\prime}(\xi)\right|$; since $\left|P^{\prime}(\xi)\right|$ should be in principle roughly equal to $H(P)$, we expect the estimation $|\xi-\alpha| \ll|P(\xi)| \cdot H(P)^{-1}$.

2000 Mathematics Subject Classification : 11J13, 11J82. 
The behaviour of the sequences $\left(w_{n}(\xi)\right)_{n \geq 1}$ and $\left(w_{n}^{*}(\xi)\right)_{n \geq 1}$ determines the localisation of $\xi$ in Mahler's and Koksma's classifications, respectively; however, the precise estimation of $w_{n}(\xi)$ and $w_{n}^{*}(\xi)$ is usually extremely difficult. The Dirichlet box principle (or, equivalently, Minkowski's theorem) readily implies that $w_{n}(\xi) \geq n$ for any positive integer $n$ and any transcendental real number $\xi$. It is a longstanding problem to decide whether the same result remains true for the functions $w_{n}^{*}$.

Conjecture (Wirsing). For any integer $n \geq 1$ and any transcendental real number $\xi$ we have $w_{n}^{*}(\xi) \geq n$.

The early paper of Wirsing [27] and the study of his conjecture, which has been up to now confirmed only for $n=1$ (this is a simple application of Dirichlet's box principle) and $n=2$ (by Davenport \& Schmidt [12]), have motivated many works. In particular, Davenport \& Schmidt [13] have investigated this question in a dual way, via simultaneous rational approximation to the successive powers of $\xi$. Among other results, they proved that if the real number $\xi$ is either transcendental, or algebraic of degree $\geq 3$, then there exist some positive constant $c$, depending only upon $\xi$, and arbitrarily large real numbers $\mathrm{X}$ such that the inequalities

$$
0<\left|x_{0}\right|<X, \quad\left|x_{0} \xi-x_{1}\right|<c X^{-(\sqrt{5}-1) / 2}, \quad\left|x_{0} \xi^{2}-x_{2}\right|<c X^{-(\sqrt{5}-1) / 2}
$$

have no integer solution $x_{0}, x_{1}, x_{2}$. This assertion is by no means surprising since the inequalities (2) define a convex body whose volume tends to 0 when $X$ tends to infinity, and one should be tempted to believe that the same conclusion holds if we replace $(\sqrt{5}-1) / 2$ by any real number larger than $1 / 2 \ldots$, but that is false. Indeed, when $\xi$ is a real number whose continued fraction expansion reproduces the structure of the Fibonacci word, Roy proved recently that there does exist an other $c$ such that (2) has an integer solution $x_{0}, x_{1}, x_{2}$ for any sufficiently large $X$ (Theorem 1.1 from [23]). The critical exponent in (2) is definitively $(\sqrt{5}-1) / 2$ for a Fibonacci continued fraction $\xi$.

The above point (2), as well as the article [3], suggests to us to introduce other exponents which implicitely appear in problems of Diophantine Approximation by algebraic numbers or algebraic integers. We define four new exponents, which naturally complement the functions $w_{n}$ and $w_{n}^{*}$.

We first denote by $\lambda_{n}(\xi)$ the exponent of simultaneous rational approximation to the numbers $\xi, \ldots, \xi^{n}$. Thus, $\lambda_{n}(\xi)$ denotes the supremum of the real numbers $\lambda$ such that the inequality

$$
\max _{1 \leq m \leq n}\left|x_{0} \xi^{m}-x_{m}\right| \leq\left|x_{0}\right|^{-\lambda}
$$

has infinitely many solutions in integers $x_{0}, \ldots, x_{n}$ with $x_{0} \neq 0$. It will also be convenient to introduce the inverse

$$
w_{n}^{\prime}(\xi)=\frac{1}{\lambda_{n}(\xi)},
$$


which is equal to $n$ for almost all real number $\xi$ (with respect to the Lebesgue measure). The three exponents $w_{n}, w_{n}^{*}$ and $\lambda_{n}$ have the common feature to be defined by the occurrence of infinitely many solutions for some set of Diophantine inequalities. We attach to them three functions defined by a condition of uniform existence of solutions and we decide to indicate this uniformity by the symbol ^ .

Definition 1.1. Let $n \geq 1$ be an integer and let $\xi$ be a real number. We denote by $\hat{w}_{n}(\xi)$ the supremum of the real numbers $w$ such that, for any sufficiently large real number $X$, the inequalities

$$
0<\left|x_{n} \xi^{n}+\ldots+x_{1} \xi+x_{0}\right| \leq X^{-w}, \quad \max _{0 \leq m \leq n}\left|x_{m}\right| \leq X
$$

have a solution in integers $x_{0}, \ldots, x_{n}$. We denote by $\hat{w}_{n}^{*}(\xi)$ the supremum of the real numbers $w$ such that, for any sufficiently large real number $X$, there exists an algebraic real number $\alpha$ with degree at most $n$ and satisfying

$$
0<|\xi-\alpha| \leq H(\alpha)^{-1} X^{-w} \quad \text { and } \quad H(\alpha) \leq X
$$

We denote by $\hat{\lambda}_{n}(\xi)$ the supremum of the real numbers $\lambda$ such that, for any sufficiently large real number $X$, the inequalities

$$
0<\left|x_{0}\right| \leq X, \quad \max _{1 \leq m \leq n}\left|x_{0} \xi^{m}-x_{m}\right| \leq X^{-\lambda}
$$

have a solution in integers $x_{0}, \ldots, x_{n}$. We also set $\hat{w}_{n}^{\prime}(\xi)=1 / \hat{\lambda}_{n}(\xi)$.

In Part 2, we establish various relations linking our six exponents. Some of them are reformulations of known results while others are new, as Theorem 2.1 below. The sequel of the article is devoted to the explicit determination of the values of these six exponents in degree $n=2$, when $\xi$ is a Sturmian continued fraction. Since these values differ from those obtained for algebraic numbers, we thus obtain a quantitative proof of the transcendence of these Sturmian continued fractions, which completes and precises the original proof in $[2]$, based on the observation that $w_{2}^{*}(\xi)>2$ for those continued fractions $\xi$. Let us indicate here that the Sturmian words comprise Fibonacci word and that we thus extend some of the results contained in $[22,23,3]$; the above point (2) being an example. The formulas obtained are stated in Part 3, our main result being Theorem 3.1. Its proof, which rests on a study of multiplicative recurrences (Lemma 4.1) and on combinatorial properties of infinite Sturmian words (Lemma 5.3), is detailled in Parts 6 and 7. It extends in a Sturmian frame the ideas developped in [23]. One of the key result is Lemma 6.1, which provides a precise estimate of the height of some quadratic numbers defined by purely periodic continued fractions. Finally, Part 8 is devoted to a discussion on the spectra of the various exponents of approximation that we have just defined. 
In a forthcoming work, we shall combine some of the ideas of the present article with the method developped by Roy $[23,24,25]$ in order to construct further examples of real numbers which are badly approximable by algebraic integers with degree $\leq 3$. We shall further establish a link with questions of inhomogeneous Diophantine Approximation.

\section{Properties of the exponents of approximation.}

This Section is devoted to an overview of general results on the six functions $w_{n}, w_{n}^{*}, \lambda_{n}, \hat{w}_{n}, \hat{w}_{n}^{*}$ and $\hat{\lambda}_{n}$. Their values are connected by various numerical inequalities. Notice that the exponents 'hat' are uniformly bounded in term of $n$. The exact determination of the upper bounds is an important problem towards the Wirsing Conjecture or connected topics, such as the approximation by algebraic integers; it is solved only for $n=1$ and $n=2$. Let us begin with some easy properties:

Proposition 2.1. For any integer $n \geq 1$ and any real number $\xi$ which is not algebraic of degree $\leq n$, we have

and

$$
n \leq \hat{w}_{n}(\xi) \leq w_{n}(\xi), \quad \frac{1}{n} \leq \hat{\lambda}_{n}(\xi) \leq \min \left\{1, \lambda_{n}(\xi)\right\}
$$

$$
1 \leq \hat{w}_{n}^{*}(\xi) \leq \min \left\{w_{n}^{*}(\xi), \hat{w}_{n}(\xi)\right\} \leq \max \left\{w_{n}^{*}(\xi), \hat{w}_{n}(\xi)\right\} \leq w_{n}(\xi)
$$

Proof. The upper bound $w_{n}^{*}(\xi) \leq w_{n}(\xi)$ is Proposition 3.2 from [10]. The same argumentation gives also that $\hat{w}_{n}^{*}(\xi) \leq \hat{w}_{n}(\xi)$. The upper bounds $\hat{w}_{n}(\xi) \leq w_{n}(\xi), \hat{w}_{n}^{*}(\xi) \leq w_{n}^{*}(\xi)$ and $\hat{\lambda}_{n}(\xi) \leq \lambda_{n}(\xi)$ are easy, while the lower bounds $\hat{w}_{n}(\xi) \geq n$ and $\hat{\lambda}_{n}(\xi) \geq 1 / n$ follow from Dirichlet's box principle (or, equivalently, Minkowski's theorem). The lower bounds $\hat{\lambda}_{1}(\xi) \geq 1, \hat{w}_{1}(\xi) \geq 1$ and $\hat{w}_{1}^{*}(\xi) \geq 1$ are direct consequences of the usual Dirichlet theorem ([10], Theorem 1.1) which asserts that for any irrational number $\xi$ and any $Q \geq 1$, there exists a rational $p / q$, with $1 \leq q \leq Q$ and $0<|\xi-p / q|<1 /(q Q)$. Since Dirichlet's box principle cannot be improved with respect to the exponent of $Q$ (cf. [14]), it turns out that we have the equalities

$$
\hat{w}_{1}(\xi)=\hat{w}_{1}^{*}(\xi)=\hat{\lambda}_{1}(\xi)=1 .
$$

Moreover, we obviously have $\hat{w}_{n}^{*}(\xi) \geq \hat{w}_{1}^{*}(\xi)=1$ and $\hat{\lambda}_{n}(\xi) \leq \hat{\lambda}_{1}(\xi)=1$.

On the other hand, when $\xi$ is a Liouville number, we have $\hat{w}_{n}^{*}(\xi)=1$ using Liouville's inequality (cf. [10], Appendix A); it follows that the lower bound $\hat{w}_{n}^{*}(\xi) \geq 1$ in (3) is optimal.

Wirsing proved the inequality

$$
w_{n}^{*}(\xi) \geq \frac{w_{n}(\xi)}{w_{n}(\xi)-n+1},
$$

for any integer $n \geq 1$ and any real number $\xi$ which is not algebraic of degree at most $n$. We can refine (4) in the following way: 
Theorem 2.1. Let $n$ be an integer $\geq 1$. The lower bounds

$$
\hat{w}_{n}^{*}(\xi) \geq \frac{w_{n}(\xi)}{w_{n}(\xi)-n+1}
$$

and

$$
w_{n}^{*}(\xi) \geq \frac{\hat{w}_{n}(\xi)}{\hat{w}_{n}(\xi)-n+1},
$$

hold for any real number $\xi$ which is not algebraic of degree $\leq n$.

Proof. We obtain the first inequality by taking again Wirsing's argumentation [27]. Let $n \geq 2$ be an integer and let $\xi$ be a real number which is either transcendental, or algebraic of degree $>n$. Let $\epsilon>0$ and set $w=w_{n}(\xi)(1+\epsilon)^{2}$. Minkowski's theorem implies that there exist a constant $c$ and, for any positive real number $H$, a non zero integer polynomial $P(X)$ of degree at most $n$ such that

$$
|P(\xi)| \leq H^{-w}, \quad|P(1)|, \ldots,|P(n-1)| \leq H \quad \text { and } \quad|P(n)| \leq c H^{w-n+1} .
$$

The definition of $w_{n}(\xi)$ and the first condition of $(7)$ show that $H(P) \gg H^{1+\epsilon}$. Consequently, $P(X)$ has some (necessarily real) root in the neighbourhood of each of the points $\xi, 1, \ldots, n-1$. Denoting by $\alpha$ the closest root to $\xi$, we get

$$
|\xi-\alpha| \gg \ll \frac{|P(\xi)|}{H(P)} \ll H(P)^{-1}\left(H^{w-n+1}\right)^{-w /(w-n+1)} \quad \text { and } \quad H(P) \ll H^{w-n+1} .
$$

Since all of this is true for any sufficiently large $H$, we get $\hat{w}_{n}^{*}(\xi) \geq w /(w-n+1)$. Selecting now $\epsilon$ arbitrarily close to 0 , we obtain (5).

In order to prove (6), we take again the argumentation of [7], reproduced in [10], which shows that if $A$ is a real number, lying in the interval $2<A<n+1$, and such that $|\xi-\alpha| \geq H(\alpha)^{-A}$ for all algebraic numbers $\alpha$ of degree $\leq n$ and sufficiently large height, then, for any $\epsilon<(n+1-A) /(A-2)$ and any sufficiently large $H$, there exists an integer polynomial $P(X)$ with height $\leq H$ satisfying $|P(\xi)| \leq H^{-n-\epsilon}$. This means that we have $\hat{w}_{n}(\xi) \geq n+\epsilon$, and next that $\hat{w}_{n}(\xi) \geq n+(n+1-A) /(A-2)$. Noticing that $w_{n}^{*}(\xi) \geq A-1$, we obtain (6).

Since $w_{n}^{*}(\xi) \geq \hat{w}_{n}^{*}(\xi)$ and $w_{n}(\xi) \geq \hat{w}_{n}(\xi)$, each of the two lower bounds from Theorem 2.1 implies the inequality (4). Moreover, the example of Liouville numbers $\xi$, for which $\hat{w}_{n}^{*}(\xi)=1$ and $w_{n}(\xi)=+\infty$, shows that the estimation (5) is best possible [17].

We indicate now some transference results relying the rational simultaneous approximation to $\xi, \ldots, \xi^{n}$ and the smallness of the linear form with coefficients $\xi, \ldots, \xi^{n}$. 
Theorem 2.2. Let $n$ be an integer $\geq 1$. We have the estimations

$$
\frac{n}{w_{n}(\xi)-n+1} \leq w_{n}^{\prime}(\xi) \leq \frac{(n-1) w_{n}(\xi)+n}{w_{n}(\xi)} \leq n
$$

and

$$
\frac{n}{\hat{w}_{n}(\xi)-n+1} \leq \hat{w}_{n}^{\prime}(\xi) \leq \frac{(n-1) \hat{w}_{n}(\xi)+n}{\hat{w}_{n}(\xi)} \leq n
$$

for any real number $\xi$ which is not algebraic of degree $\leq n$.

Proof. The inequalities (8) follow direcly from Khintchine's transference principle (cf. Theorem B.5 from [10]), whose proof shows in fact that (9) is also true. [

We know the behaviour of these six functions for almost all real numbers $\xi$, and also their values for algebraic real numbers $\xi$.

Theorem 2.3. For almost all (with respect to Lebesgue measure) real number $\xi$ and any positive integer $n$, we have

$$
w_{n}(\xi)=w_{n}^{*}(\xi)=w_{n}^{\prime}(\xi)=\hat{w}_{n}(\xi)=\hat{w}_{n}^{\prime}(\xi)=\hat{w}_{n}^{*}(\xi)=n
$$

Proof. A result due to Sprindžuk [26] asserts that $w_{n}(\xi)$ is equal to $n$ for almost all $\xi$. Thank to Proposition 2.1, to (4) and to Theorem 2.2, we obtain that $w_{n}^{*}(\xi)=\hat{w}_{n}^{*}(\xi)=w_{n}^{\prime}(\xi)=n$ for almost all $\xi$. Besides, Bugeaud [8] proved that $\hat{w}_{n}(\xi)=n$ (and therefore $\hat{w}_{n}^{\prime}(\xi)=n$, by (9)) for almost all $\xi$. [

Theorem 2.4. Let $\xi$ be a real algebraic number of degree $d \geq 2$ and let $n$ be a positive integer. We have

$$
w_{n}(\xi)=w_{n}^{*}(\xi)=w_{n}^{\prime}(\xi)=\hat{w}_{n}(\xi)=\hat{w}_{n}^{\prime}(\xi)=\hat{w}_{n}^{*}(\xi)=\min \{n, d-1\}
$$

Proof. This is a straightforward consequence of Schmidt's Subspace Theorem. We omit the details.

Davenport \& Schmidt [13] have investigated the approximation to a real number by algebraic integers, using a dual approch which may also be applied to Wirsing's Conjecture. Their results have been recently improved by Laurent [18]. For a positive real number $x$, we denote by $\lceil x\rceil$ the smallest integer $\geq x$. The following statement translates the main results of [13] and of [18] in terms of exponents: 
Theorem 2.5. For any integer $n \geq 1$ and any transcendental real number $\xi$, we have

$$
w_{n}^{*}(\xi) \geq \hat{w}_{n}^{\prime}(\xi) \geq\lceil n / 2\rceil \text { and } \hat{w}_{n}(\xi) \leq 2 n-1 .
$$

Notice that the estimation

$$
w_{n}^{*}(\xi) \geq \hat{w}_{n}^{\prime}(\xi) \geq \frac{n}{\hat{w}_{n}(\xi)-n+1},
$$

which follows from (9) and from Theorem 2.5, is only slightly weaker than the inequality (6).

For $n=2$, recent results of Roy [22, 23] and Arbour \& Roy [3] give us the extremal values of the functions $\hat{w}_{2}, \hat{w}_{2}^{\prime}$ and $\hat{w}_{2}^{*}$.

Theorem 2.6. In degree $n=2$, the refined inequalities

$$
\hat{w}_{2}^{\prime}(\xi) \geq \frac{1+\sqrt{5}}{2}, \quad \hat{w}_{2}(\xi) \leq \frac{3+\sqrt{5}}{2} \quad \text { and } \quad \hat{w}_{2}^{*}(\xi) \leq \frac{3+\sqrt{5}}{2}
$$

hold for any real number $\xi$ which is neither rational, nor quadratic. Moreover these inequalties are best possible.

In Theorem 2.6, the equalities are reached for instance with the number

$$
\xi=[0 ; 1,2,1,1,2,1,2, \ldots],
$$

whose sequence of partial quotients reproduces the letters of the Fibonacci word, up to the initial term. This word is an example of Sturmian word which will be considered in Part 3. It corresponds to the particular case of a constant sequence $\left(s_{k}\right)_{k \geq 1}$ equal to one.

\section{Sturmian continued fractions.}

Our goal is to construct other examples of real numbers $\xi$ (necessarily transcendental) for which one can compute the values at $\xi$ of the six functions $w_{2}, \ldots, \hat{w}_{2}^{*}$, all these values being distinct from 2 . We show that typical Sturmian continued fractions share this property.

Let $\left(s_{k}\right)_{k \geq 1}$ be a sequence of integers $\geq 1$ and let $\{a, b\}$ be an alphabet with two letters. We denote by $\{a, b\}^{*}$ the monoïd of finite words on this alphabet and we define inductively a sequence of words $\left(m_{k}\right)_{k \geq 0}$ belonging to $\{a, b\}^{*}$ by the formulas

$$
m_{0}=b, \quad m_{1}=b^{s_{1}-1} a \quad \text { and } \quad m_{k+1}=m_{k}^{s_{k+1}} m_{k-1} \quad(k \geq 1) .
$$

This sequence converges (for the product of the discrete topologies) in the completion $\{a, b\}^{*} \cup\{a, b\}^{\mathbf{N}}$ to the infinite word

$$
m_{\varphi}:=\lim _{k \rightarrow \infty} m_{k}=b^{s_{1}-1} a \ldots,
$$


which is usually called the Sturmian characteritic word of angle (or of 'slope')

$$
\varphi:=\left[0 ; s_{1}, s_{2}, s_{3}, \ldots\right]
$$

constructed on the alphabet $\{a, b\}$.

The characters $a$ and $b$ will indicate either the letters $\{a, b\}$ of the alphabet, or distinct positive integers, according to the context. Denote by $\xi_{\varphi}=\left[0 ; m_{\varphi}\right]$ the real number whose partial quotients are succesively 0 , and the letters of the infinite word $m_{\varphi}$, and set

$$
\sigma_{\varphi}=\liminf _{k}\left[0 ; s_{k}, s_{k-1}, \ldots, s_{1}\right]=\frac{1}{\lim \sup _{k}\left[s_{k} ; s_{k-1}, \ldots, s_{1}\right]} .
$$

In order to shorten the notations, we write $\sigma$ instead of $\sigma_{\varphi} .\left(^{*}\right)$

The real number $\xi$ defined after Theorem 2.6 is equal to the number $\xi_{(\sqrt{5}-1) / 2}$ written on the alphabet $\{1,2\}$. The corresponding Fibonacci word $m_{(\sqrt{5}-1) / 2}=12112121 \ldots$ satisfies two important properties. Firstly, it contains many initial factors of the shape $u u u^{\prime}$, where $u$ is a word and $u^{\prime}$ is some prefix of $u$. Since the quadratic real numbers are those whose continued fraction expansion is ultimately periodic, it follows that $\xi_{(\sqrt{5}-1) / 2}$ has a lot of very good quadratic approximations. Secondly, the word $m_{(\sqrt{5}-1) / 2}$ contains many palindromic prefixes. As was observed by Roy [22], this last property enables us to construct rational simultaneous approximations to $\xi_{(\sqrt{5}-1) / 2}$ and its square. It turns out that similar properties also hold true for more general Sturmian words, as we shall see in Part 5.

Here is our main result.

Theorem 3.1. Let $a$ and $b$ be distinct positive integers. We have

$$
\begin{gathered}
\lambda_{2}\left(\xi_{\varphi}\right)=1, \quad w_{2}\left(\xi_{\varphi}\right)=w_{2}^{*}\left(\xi_{\varphi}\right)=1+\frac{2}{\sigma} \\
\hat{\lambda}_{2}\left(\xi_{\varphi}\right)=\frac{1+\sigma}{2+\sigma}=\frac{1}{2}+\frac{\sigma}{2(2+\sigma)} \quad \text { and } \quad \hat{w}_{2}\left(\xi_{\varphi}\right)=\hat{w}_{2}^{*}\left(\xi_{\varphi}\right)=2+\sigma .
\end{gathered}
$$

It follows immediately from Theorem 2.4 that $\xi_{\varphi}$ is a transcendental number. This result was originally established in [2].

The proof of Theorem 3.1 requests several steps. We first have to control the growth of the denominators of some convergents of $\xi_{\varphi}$ (Lemma 4.1). Next we establish combinatorial properties of the word $m_{\varphi}$ (Lemma 5.3). Part 6 is devoted to the computation of the exponents $w_{2}, w_{2}^{*}, \hat{w}_{2}$ et $\hat{w}_{2}^{*}$, while $\lambda_{2}$ and $\hat{\lambda}_{2}$ are treated in Part 7 .

We briefly indicate two immediate consequences of Theorem 3.1.

$\left(^{*}\right)$ It is readily seen that the quantity $\sigma$ does not depend upon the initial values of the sequence $\left(s_{k}\right)$. Thus, in the definition of $\sigma$, the use of the reverse continued fraction $\left[s_{k}, \ldots, s_{1}\right]$, ending exactly by $s_{1}, s_{2}, \ldots$, is purely conventional. 
Corollary 3.1. For any positive integer $d$, there exists a real number $\xi$ satisfying

$$
\begin{gathered}
w_{2}(\xi)=w_{2}^{*}(\xi)=\sqrt{d^{2}+4}+d+1 \\
\hat{\lambda}_{2}(\xi)=\frac{\sqrt{d^{2}+4}+2-d}{\sqrt{d^{2}+4}+4-d} \text { and } \quad \hat{w}_{2}(\xi)=\hat{w}_{2}^{*}(\xi)=2+\frac{\sqrt{d^{2}+4}-d}{2} .
\end{gathered}
$$

Proof. Apply the theorem to the constant sequence $s_{k}$ equal to $d$, for which we have $\sigma=2 /\left(d+\sqrt{d^{2}+4}\right)$

Corollary 3.1 gives us an explicit sequence of numbers $>2($ resp. $>1 / 2)$ belonging to the spectrum of the function $\hat{w}_{2}$ (resp. $\left.\hat{\lambda}_{2}\right)$. More results on these spectra are obtained in Part 8.

Corollary 3.2. There exists a continuous set of real numbers $\xi$ satisfying

$$
\hat{w}_{2}^{\prime}(\xi)<2, \quad \hat{w}_{2}(\xi)>2 \text { and } \hat{w}_{2}^{*}(\xi)>2 .
$$

It proceeds from Theorem 2.3 that the set of real numbers $\xi$ for which $\hat{w}_{2}^{*}(\xi)$ is larger than 2 has a Lebesgue measure equal to zero. This set is not countable, and it is an interesting problem to compute its Hausdorff dimension.

To conclude this Section, let us point out that the quantity $\sigma^{-1}$ appears further in measures of irrationality of some series associated to Sturmian sequences, as was indicated to us by Boris Adamczewski. Denote by $\left(\ell_{j}\right)_{j \geq 1}$ the sequence formed by the letters of the word $m_{\varphi}$ written on the alphabet $\{a, b\}$. If we choose the values $a=1$ and $b=0$, the irrationality measure $w_{1}(\beta)$ of the number

$$
\beta=\sum_{j \geq 1} \frac{\ell_{j}}{2^{j}}
$$

is exactly equal to $\lim \sup _{k}\left[s_{k} ; s_{k-1}, \ldots, s_{1}\right]$. This result has been established by Davison [15] for $\varphi=(\sqrt{5}-1) / 2$ and by Adams \& Davison [1] in the general case (they do not state it, but the result is implicitely contained in their work, as well as in an article of Queffélec $[20])$.

\section{Multiplicative recursions.}

Let $\left(X_{n}\right)_{n \geq 0}$ be a sequence of positive real numbers and let $\left(s_{n}\right)_{n \geq 1}$ be a sequence of positive integers. We suppose that the sequence $\left(X_{n}\right)_{n \geq 0}$ tends to infinity and that there exists $c \geq 1$ such that the inequalities

$$
c^{-s_{n+1}} X_{n}^{s_{n+1}} X_{n-1} \leq X_{n+1} \leq c^{s_{n+1}} X_{n}^{s_{n+1}} X_{n-1},
$$

hold for any $n \geq 1$. The aim of this Section is to estimate the growth of the sequence $\left(X_{n}\right)_{n \geq 0}$. 
Lemma 4.1. For any $n \geq 0$, define $\eta_{n}$ by $X_{n+1}=X_{n}^{\eta_{n}}$. Then, we have

$$
\eta_{n}=\left[s_{n+1} ; s_{n}, \ldots, s_{1}\right](1+o(1))
$$

when $n$ tends to infinity.

Proof. Using the right upper bound of (10), we obtain successively

$$
X_{n} \leq c^{s_{n}} X_{n-1}^{s_{n}} X_{n-2} \leq c^{s_{n} s_{n-1}+s_{n}} X_{n-2}^{s_{n} s_{n-1}+1} X_{n-3}^{s_{n}} \leq \cdots
$$

Here occurs the sequence of integers $q_{k}=q_{k, n}$ defined for $k=-1, \ldots, n$ by

$$
q_{-1}=0, \quad q_{0}=1, \quad q_{1}=s_{n}, \quad q_{2}=s_{n} s_{n-1}+1, \ldots
$$

and satisfying the binary recurrent relation $q_{k+1}=s_{n-k} q_{k}+q_{k-1}$ for $0 \leq k \leq n-1$. Let us check by induction on $k=0, \ldots, n-1$ the upper bound

$$
X_{n} \leq c^{q_{k}+q_{k-1}-1} X_{n-k}^{q_{k}} X_{n-k-1}^{q_{k-1}}
$$

The estimation is obvious for $k=0$. Suppose that it holds up to the rank $k$; then (10) gives

$$
X_{n} \leq c^{q_{k}+q_{k-1}-1}\left(c^{s_{n-k}} X_{n-k-1}^{s_{n-k}} X_{n-k-2}\right)^{q_{k}} X_{n-k-1}^{q_{k-1}}=c^{q_{k+1}+q_{k}-1} X_{n-k-1}^{q_{k+1}} X_{n-k-2}^{q_{k}}
$$

from which follows the upper bound at the rank $k+1$. Using the left lower bound from (10), we obtain similarly

$$
X_{n} \geq c^{-q_{k}-q_{k-1}+1} X_{n-k}^{q_{k}} X_{n-k-1}^{q_{k-1}}
$$

We shall compare $X_{n+1}$ with $X_{n}$ using both estimations. To that purpose, let us first prove the formula

$$
\left[s_{n+1} ; \ldots, s_{n+1-k}\right]=\frac{q_{k+1, n+1}}{q_{k, n}},
$$

that is given by the theory of continued fractions. The recurrent relations

$$
q_{j+1, n+1}=s_{n+1-j} q_{j, n+1}+q_{j-1, n+1} \quad \text { and } \quad q_{j, n}=s_{n+1-j} q_{j-1, n}+q_{j-2, n},
$$

are satisfied for $1 \leq j \leq n$ and imply the matrix relation

$$
\left(\begin{array}{cc}
q_{k+1, n+1} & q_{k, n+1} \\
q_{k, n} & q_{k-1, n}
\end{array}\right)=\left(\begin{array}{cc}
s_{n+1} & 1 \\
1 & 0
\end{array}\right) \cdots\left(\begin{array}{cc}
s_{n+1-k} & 1 \\
1 & 0
\end{array}\right),
$$

from which follows the expected formula. 
We have established the estimations

$$
X_{n} \geq\left(c^{-1} X_{k}\right)^{q_{n-k, n}}\left(c^{-1} X_{k-1}\right)^{q_{n-k-1, n}} \quad \text { and } \quad X_{n+1} \leq\left(c X_{k}\right)^{q_{n-k+1, n+1}}\left(c X_{k-1}\right)^{q_{n-k, n+1}}
$$

for any $k=0, \ldots, n$. Let $\epsilon$ be a positive real number and let $k$ be a sufficiently large integer such that $c \leq \min \left\{X_{k}^{\epsilon}, X_{k-1}^{\epsilon}\right\}$. Then, we get

$$
\begin{aligned}
X_{n+1} & \leq\left(c^{-1} X_{k}\right)^{\frac{1+\epsilon}{1-\epsilon} q_{n-k+1, n+1}}\left(c^{-1} X_{k-1}\right)^{\frac{1+\epsilon}{1-\epsilon} q_{n-k, n+1}} \\
& \leq X_{n}^{\frac{1+\epsilon}{1-\epsilon} \max }\left\{\frac{q_{n-k+1, n+1}}{q_{n-k, n}}, \frac{q_{n-k, n+1}}{q_{n-k-1, n}}\right\}
\end{aligned}
$$

Since

$$
\frac{q_{n-k+1, n+1}}{q_{n-k, n}}=\left[s_{n+1} ; \ldots, s_{k+1}\right]=\left[s_{n+1} ; \ldots, s_{1}\right]+o_{k}(1)
$$

and

$$
\frac{q_{n-k, n+1}}{q_{n-k-1, n}}=\left[s_{n+1} ; \ldots, s_{k+2}\right]=\left[s_{n+1} ; \ldots, s_{1}\right]+o_{k}(1)
$$

when $n$ tends to infinity, we obtain

$$
\eta_{n} \leq\left[s_{n+1} ; \ldots, s_{1}\right](1+o(1))
$$

The lower bound for $\eta_{n}$ is proved in the same way.

\section{Combinatorial properties of Sturmian words.}

We take again the notations of Part 3, where $m_{\varphi}$ denotes the limit of the sequence of words $\left(m_{k}\right)_{k \geq 0}$ defined by

$$
m_{0}=b, \quad m_{1}=b^{s_{1}-1} a \quad \text { and } \quad m_{k+1}=m_{k}^{s_{k+1}} m_{k-1}
$$

for any $k \geq 1$. In this Section, we prove two combinatorial properties satisfied by the infinite word $m_{\varphi}$, namely the existence of many (large) initial powers and that of many initial palindromes.

For any word $u$ having at least two letters, we denote by $u^{\prime}$ the word $u$ deprived of its two last letters. Thus, when $k \geq 2$, we write $m_{k}^{\prime}$ for the word $m_{k}$ deprived of its two last letters $f_{k}=a b$ if $k$ is even and $f_{k}=b a$ if $k$ is odd.

We start with a classical result on Sturmian words. 
Lemma 5.1. For any integer $k \geq 3$, we have $m_{k} m_{k-1}^{\prime}=m_{k-1} m_{k}^{\prime}$ and the word $m_{k+2}$ begins by $m_{k}^{1+s_{k+1}} m_{k-1}^{\prime} f_{k}$.

Proof. This is Proposition 1 from [2]. The second assertion is proved in this article, but an assertion slightly weaker is stated. $\square$

Lemma 5.2. For any integer $k \geq 3$, the two words $m_{\varphi}$ and $m_{k} m_{k} \ldots$ have exactly for common initial part the word $m_{k}^{1+s_{k+1}} m_{k-1}^{\prime}$.

Proof. It follows from Lemma 5.1 that $m_{k}^{1+s_{k+1}} m_{k-1}^{\prime} f_{k}$ is a prefix of the word $m_{\varphi}$, while the periodic word $m_{k} m_{k} \ldots$ begins with $m_{k}^{1+s_{k+1}} m_{k-1}=m_{k}^{1+s_{k+1}} m_{k-1}^{\prime} f_{k-1}$. Since $\left(f_{k-1}, f_{k}\right)=(a b, b a)$ or $(b a, a b)$, the lemma is established.

To any word $m$ we associate the sequence, ordered by inclusion, of prefixes of this word which are palindromes. The following result describes some subsequence extracted from this sequence in the case of the Sturmian word $m_{\varphi}$. It should be interesting to know whether we obtain in this way all palindromic prefixes of the word $m_{\varphi}$ up to a finite number of exceptions, which seems to be experimentally the case. By convention, the empty word is viewed as a palindrome.

Lemma 5.3. The sequence of words

$$
\left(m_{k}^{t} m_{k-1}\right)^{\prime}, \quad \text { où } \quad k \geq 1 \quad \text { and } \quad 1 \leq t \leq s_{k+1},
$$

and where the indices $(k, t)$ are lexicographically ordered, makes up an increasing sequence of prefixes of the word $m_{\varphi}$ which are palindromes.

Proof. It will be convenient to state the relation of commutation $m_{k} m_{k-1}^{\prime}=m_{k-1} m_{k}^{\prime}$ of Lemma 5.1 in the equivalent form

$$
\left(m_{k} m_{k-1}\right)^{\prime}=\left(m_{k-1} m_{k}\right)^{\prime}
$$

which has the advantage to be valid for $k=1$ and $k=2$.

We show by induction on the rank of the couples $(k, t)$ lexicographically ordered that the prefixes $\left(m_{k}^{t} m_{k-1}\right)^{\prime}$ are palindromes. When $k=1$ and $t \geq 1$, or $k=2$ and $t=1$, the words

$$
\begin{aligned}
& \left(m_{1}^{t} m_{0}\right)^{\prime}=\left(b^{s_{1}-1} a\right)^{t-1} b^{s_{1}-1}, \\
& \left(m_{2} m_{1}\right)^{\prime}=\left(b^{s_{1}-1} a\right)^{s_{2}} b^{s_{1}-1} \\
& \left(m_{2}^{2} m_{1}\right)^{\prime}=b^{s_{1}-1}\left(a b^{s_{1}-1}\right)^{s_{2}-1} a b^{s_{1}} a\left(b^{s_{1}-1} a\right)^{s_{2}-1} b^{s_{1}-1}
\end{aligned}
$$


are clearly palindromes. Suppose now that the recurrence hypothesis is satisfied up to the rank $(k, t)$ with $(k, t) \geq(2,2)$. In order to show that

$$
\left(m_{k}^{t+1} m_{k-1}\right)^{\prime}=m_{k}^{t}\left(m_{k} m_{k-1}\right)^{\prime}
$$

is a palindrome, let us write

$$
\begin{aligned}
m_{k}^{t}\left(m_{k} m_{k-1}\right)^{\prime} & =\left(m_{k-1}^{s_{k}} m_{k-2}\right)^{\prime} f_{k} m_{k}^{t-1} m_{k-1}^{s_{k}}\left(m_{k-2} m_{k-1}\right)^{\prime} \\
& =\left(m_{k-1}^{s_{k}} m_{k-2}\right)^{\prime} f_{k}\left(m_{k}^{t-1} m_{k-1}\right)^{\prime} f_{k-1} m_{k-1}^{s_{k}-1}\left(m_{k-1} m_{k-2}\right)^{\prime} \\
& =\left(m_{k-1}^{s_{k}} m_{k-2}\right)^{\prime} f_{k}\left(m_{k}^{t-1} m_{k-1}\right)^{\prime} f_{k-1}\left(m_{k-1}^{s_{k}} m_{k-2}\right)^{\prime} .
\end{aligned}
$$

Thank to the recurrence hypothesis, the words $\left(m_{k-1}^{s_{k}} m_{k-2}\right)^{\prime}$ and $\left(m_{k}^{t-1} m_{k-1}\right)^{\prime}$ are palindromes, and $f_{k-1}$ is the mirror of $f_{k}$. We deduce that the word $\left(m_{k}^{t+1} m_{k-1}\right)^{\prime}$ is also a palindrome.

When $t=s_{k+1}$, the successor of $\left(m_{k}^{t} m_{k-1}\right)^{\prime}=m_{k+1}^{\prime}$ is $m_{k+1} m_{k}^{\prime}=m_{k+1}^{\prime} f_{k+1} m_{k}^{\prime}$. The mirror of this last word is then

$$
m_{k}^{\prime} f_{k} m_{k+1}^{\prime}=m_{k} m_{k+1}^{\prime}=m_{k+1} m_{k}^{\prime}
$$

since the words $m_{k+1}^{\prime}$ are $m_{k}^{\prime}$ are palindromes, again by the recurrence hypothesis.

\section{The exponents $w_{2}, w_{2}^{*}, \hat{w}_{2}$ and $\hat{w}_{2}^{*}$.}

We keep the notations of Part 5 and denote by $\alpha_{k}$ the quadratic number whose continued fraction expansion is purely periodic with period $m_{k}$ :

$$
\alpha_{k}=\left[0 ; m_{k}, m_{k}, \ldots\right]
$$

Let $M_{k}$ and $M_{k}^{\prime}$ be the $2 \times 2$ matrices associated to the words $m_{k}$ and $m_{k}^{\prime}$ by the rule

$$
M=\prod_{i=1}^{q}\left(\begin{array}{cc}
\ell_{i} & 1 \\
1 & 0
\end{array}\right)
$$

if the word $m$ is written $\ell_{1} \ldots \ell_{q}$ with letters $\ell_{i} \in\{a, b\}$. Recall that $a$ and $b$ indicate at the same time letters and distinct positive integers. Any multiplicative identity on words translates into the same identity between matrices $M$. In particular, the recurrence relation $m_{k+1}=m_{k}^{s_{k+1}} m_{k-1}$ implies that

$$
M_{k+1}=M_{k}^{s_{k+1}} M_{k-1}
$$

If $M$ is any matrix with integer coefficients, we denote by $H(M)$ its height, that is, the maximum of the absolute values of its coefficients. Let $M$ and $M^{\prime}$ be $2 \times 2$ matrices with 
positive coefficients. Then, the height of their product $M M^{\prime}$ clearly satisfies the relation of quasi-multiplicativity

$$
H(M) H\left(M^{\prime}\right) \leq H\left(M M^{\prime}\right) \leq 2 H(M) H\left(M^{\prime}\right) .
$$

Let

$$
X_{k}=H\left(M_{k}\right)
$$

denote the height of the matrix $M_{k}$. By iterated application of the inequalities (11), we see that the hypothesis (10) from Part 4 is satisfied here with $c=2$. Moreover, the sequence $X_{k}$ tends to infinity since the coefficients of $M_{k}$ are numerators and denominators of distinct convergents of $\xi_{\varphi}$. In fact, it is easily seen that the sequence $X_{k}$ increases at least as fast as a double exponential in $k$, but we shall not need this point. Consequently, Lemma 4.1 can be applied to the sequence $X_{k}$.

In the sequel, we write $F \gg G$ to signify that there exists a positive real number $\kappa$ depending only on $a, b$ and on the sequence $s_{k}$, such that we have $F(*) \geq \kappa G(*)$ for all the values of the data $*$ considered in some statement. The notation $F \gg \ll G$ signifies naturally that we simultaneously have $F \gg G$ and $F \ll G$.

The following lemma shows that the height of the quadratic number $\alpha_{k}$ has the same magnitude as $X_{k}$.

Lemma 6.1. We have $H\left(\alpha_{k}\right) \gg \ll X_{k}$. Moreover, the leading coefficient of the minimal polynomial $P_{\alpha_{k}}$ of $\alpha_{k}$ over $\mathbf{Z}$ is $\gg X_{k}$ and the conjugate $\alpha_{k}^{\prime}$ of $\alpha_{k}$ satisfies $\left|\alpha_{k}^{\prime}-\alpha_{k}\right| \gg 1$.

Proof. The number $1 / \alpha_{k}$ is a fixed point of the homography associated to the matrix $M_{k}$. The upper bound $H\left(\alpha_{k}\right) \ll X_{k}$ is then obvious. The lower bound $H\left(\alpha_{k}\right) \gg X_{k}$ needs more elaborate arguments. Notice that

$$
M_{k}=M_{k}^{\prime} F_{k}
$$

with

$F_{k}=\left(\begin{array}{ll}a & 1 \\ 1 & 0\end{array}\right)\left(\begin{array}{ll}b & 1 \\ 1 & 0\end{array}\right)=\left(\begin{array}{cc}a b+1 & a \\ b & 1\end{array}\right) \quad$ or $\quad F_{k}=\left(\begin{array}{ll}b & 1 \\ 1 & 0\end{array}\right)\left(\begin{array}{ll}a & 1 \\ 1 & 0\end{array}\right)=\left(\begin{array}{cc}a b+1 & b \\ a & 1\end{array}\right)$

according to the parity of $k$. Set $F_{k}=\left(\begin{array}{ll}f_{0} & f_{1} \\ f_{2} & f_{3}\end{array}\right)$ and observe that the matrix $M_{k}^{\prime}=$ $\left(\begin{array}{ll}x_{0} & x_{1} \\ x_{1} & x_{2}\end{array}\right)$ is symmetrical, since $m_{k}^{\prime}$ is a palindrome. The quadratic number $\alpha_{k}$ is then a root of the trinomial

$$
\left(f_{1} x_{0}+f_{3} x_{1}\right) T^{2}+\left(f_{0} x_{0}+\left(f_{2}-f_{1}\right) x_{1}-f_{3} x_{2}\right) T-f_{0} x_{1}-f_{2} x_{2} .
$$


The coefficients of the three linear forms in $x_{0}, x_{1}, x_{2}$ defined by the three coefficients of the above trinomial form a matrix whose determinant

$$
\left(f_{2}-f_{1}\right)\left(f_{0} f_{3}-f_{1} f_{2}\right)= \pm(a-b)
$$

is non zero, because $a$ and $b$ are distinct. Since the integers $x_{0}, x_{1}, x_{2}$ are relatively prime, the gcd of the coefficients of this trinomial is bounded. It follows that the leading coefficient of the polynomial $P_{\alpha_{k}}$ is obtained by dividing $f_{1} x_{0}+f_{3} x_{1}$ (which is $\gg X_{k}$ ) by this gcd and that

$$
H\left(\alpha_{k}\right) \gg H\left(M_{k}^{\prime}\right) \gg H\left(M_{k}\right)=X_{k}
$$

Notice finally that the discriminant of the trinomial is

$$
\geq\left(f_{1} x_{0}+f_{3} x_{1}\right)\left(f_{0} x_{1}+f_{3} x_{1}\right) \gg X_{k}^{2}
$$

since the coefficients of the matrices $M_{k}^{\prime}$ and $F_{k}$ are all $\geq 0$. It follows that $\left|\alpha_{k}^{\prime}-\alpha_{k}\right| \gg 1$. An other way to prove this result rests on the observation that the continued fraction expansion of $1 / \alpha_{k}$ is purely periodic and therefore, by a classical result of Galois (cf. for example [21], Theorem 3, page 45), its conjugate belongs to the interval $[0,1]$.

We deduce now from Lemmas 5.2 and 6.1 the exact values of $w_{2}\left(\xi_{\varphi}\right)$ and of $w_{2}^{*}\left(\xi_{\varphi}\right)$.

Corollary 6.1. We have $w_{2}\left(\xi_{\varphi}\right)=w_{2}^{*}\left(\xi_{\varphi}\right)=1+2 / \sigma$.

Proof. It follows from Lemma 5.2 that the words $m$ and $m_{k} m_{k} \ldots$ have $m_{k}^{1+s_{k+1}} m_{k-1}^{\prime}$ as a common prefix. Thus the partial quotients of the numbers $\xi_{\varphi}$ and $\alpha_{k}$ coincide first with 0 , next with the letters of the word $m_{k}^{1+s_{k+1}} m_{k-1}^{\prime}=m_{k} m_{k+1}^{\prime}$, and differ just after. Let $N$ be the number of letters of the word $m_{k} m_{k+1}^{\prime}$, and let

$$
\frac{p_{0}}{q_{0}}=0, \frac{p_{1}}{q_{1}}, \ldots, \frac{p_{N}}{q_{N}}
$$

be the $N+1$ first convergents which are common to both real numbers $\xi_{\varphi}$ and $\alpha_{k}$. Then, the theory of continued fractions shows us that

$$
M_{k} M_{k+1}^{\prime}=\left(\begin{array}{ll}
q_{N} & q_{N-1} \\
p_{N} & p_{N-1}
\end{array}\right)
$$

and that we have the estimate

$$
\frac{1}{q_{N}^{2}} \ll\left|\xi_{\varphi}-\alpha_{k}\right| \leq \frac{1}{q_{N}^{2}}
$$


the lower bound coming from the fact that the partial quotients of the numbers $\xi_{\varphi}$ and $\alpha_{k}$ are bounded, since they are equal to $a$ or to $b$. Thank to Lemmas 4.1 and 6.1, we thus obtain the inequalities

$$
\left|\xi_{\varphi}-\alpha_{k}\right| \gg \ll H\left(M_{k} M_{k+1}^{\prime}\right)^{-2} \gg \ll\left(X_{k} X_{k+1}\right)^{-2} \gg \ll H\left(\alpha_{k}\right)^{-2-2 \eta_{k}}
$$

from which follows the lower bound $w_{2}^{*}\left(\xi_{\varphi}\right) \geq 1+2 / \sigma$. In particular, we have $w_{2}^{*}\left(\xi_{\varphi}\right)=+\infty$ whenever the sequence $\left(s_{k}\right)_{k \geq 1}$ is unbounded. If this sequence is bounded, we proceed in the following way to get an upper bound for $w_{2}^{*}\left(\xi_{\varphi}\right)$.

Let $\alpha$ be a real algebraic number of degree $\leq 2$. Let $k$ be the integer defined by the inequalities

$$
H\left(\alpha_{k-1}\right) \leq H(\alpha)<H\left(\alpha_{k}\right)
$$

Liouville's inequality (cf. [10], Appendix A) gives the lower bound

$$
\left|\alpha_{k}-\alpha\right| \gg H(\alpha)^{-2} H\left(\alpha_{k}\right)^{-2} \gg H\left(\alpha_{k}\right)^{-4}
$$

while (12) implies that

$$
\left|\xi_{\varphi}-\alpha_{k}\right| \ll H\left(\alpha_{k}\right)^{-2-2 \eta_{k}} \ll H\left(\alpha_{k}\right)^{-4-\epsilon}
$$

for some positive real number $\epsilon$ depending on the maximal value of the $s_{k}$. Then, we deduce from the triangle inequality that

$$
\left|\xi_{\varphi}-\alpha\right| \geq\left|\alpha_{k}-\alpha\right|-\left|\xi_{\varphi}-\alpha_{k}\right| \gg H(\alpha)^{-2} H\left(\alpha_{k}\right)^{-2} \gg H(\alpha)^{-2-2 \eta_{k-1}}
$$

which implies that $w_{2}^{*}\left(\xi_{\varphi}\right) \leq 1+2 / \sigma$.

In order to show that $w_{2}\left(\xi_{\varphi}\right)=1+2 / \sigma$, one proceeds in a similar way, evaluating any integer polynomial $P(X)$ of degree $\leq 2$ at the point $\alpha_{k}$, where the index $k$ is defined by $H\left(\alpha_{k-1}\right) \leq H(P)<H\left(\alpha_{k}\right)$. The details are left to the reader. $\square$

The quadratic approximations furnished by Lemma 6.1 are sufficiently dense to determine exactly the values of the exponents $\hat{w}_{2}\left(\xi_{\varphi}\right)$ and $\hat{w}_{2}^{*}\left(\xi_{\varphi}\right)$.

Corollary 6.2. We have $\hat{w}_{2}\left(\xi_{\varphi}\right)=\hat{w}_{2}^{*}\left(\xi_{\varphi}\right)=2+\sigma$.

Proof. Lemma 4.1, together with (12) rewritten in the form

$$
\left|\xi_{\varphi}-\alpha_{k}\right| \gg \ll\left(X_{k} X_{k+1}\right)^{-2} \gg \ll H\left(\alpha_{k}\right)^{-1} H\left(\alpha_{k+1}\right)^{-\left(2+\frac{1}{\eta_{k}}\right)}
$$


implies the lower bound

$$
\hat{w}_{2}^{*}\left(\xi_{\varphi}\right) \geq 2+\liminf _{k}\left(\left[0 ; s_{k}, s_{k-1}, \ldots, s_{1}\right]\right)=2+\sigma .
$$

Suppose now that

$$
\hat{w}_{2}\left(\xi_{\varphi}\right)>2+\sigma .
$$

We shall reach a contradiction starting with an integer $k$ and real numbers $\epsilon, w$, such that

$$
\hat{w}_{2}\left(\xi_{\varphi}\right)>w \geq 2+\frac{1}{\eta_{k}}+\epsilon .
$$

This will imply $\hat{w}_{2}\left(\xi_{\varphi}\right) \leq 2+\sigma$ and therefore

$$
\hat{w}_{2}^{*}\left(\xi_{\varphi}\right)=\hat{w}_{2}\left(\xi_{\varphi}\right)=2+\sigma .
$$

By assumption, we may suppose $k$ arbitrarily large with $w$ and $\epsilon$ fixed. Set $X=\rho X_{k+1}$ for some small constant $\rho$ (independent of $k$ ). Thus, there exists a non zero integer polynomial $P(X)$ of degree $\leq 2$ such that

$$
H(P) \leq X \quad \text { and } \quad\left|P\left(\xi_{\varphi}\right)\right| \leq X^{-w}
$$

Let us prove that $P\left(\alpha_{k}\right)=0$. Otherwise, Liouville's inequality, together with the triangle inequality and Rolle's Lemma applied to $\left|P\left(\alpha_{k}\right)-P\left(\xi_{\varphi}\right)\right|$, gives us the inequalities

$$
\begin{aligned}
\rho^{-1} X_{k+1}^{-1} X_{k}^{-2} \ll H(P)^{-1} H\left(\alpha_{k}\right)^{-2} & \ll\left|P\left(\alpha_{k}\right)\right| \\
& \ll \max \left\{H(P)\left(X_{k} X_{k+1}\right)^{-2},\left(\rho X_{k+1}\right)^{-w}\right\} .
\end{aligned}
$$

If the maximum on the right hand side of (15) is reached by the second term, we obtain

$$
H(P) \gg\left(\rho X_{k+1}\right)^{w} H\left(\alpha_{k}\right)^{-2} \gg \rho^{w} X_{k+1}^{2+\frac{1}{\eta_{k}}+\epsilon} X_{k}^{-2}=\rho^{w} X_{k+1}^{2-\frac{1}{\eta_{k}}+\epsilon} .
$$

Bounding roughly from below $\eta_{k} \geq 1-o(1)$ by Lemma 4.1, the above lower bound for $H(P)$ then contradicts its upper bound $H(P) \leq \rho X_{k+1}$, when $k$ is large enough. Therefore, the maximum in (15) is equal to $H(P) X_{k}^{-2} X_{k+1}^{-2}$. Then (15) implies that $H(P) \gg \rho^{-1} X_{k+1}$, in contradiction with the upper bound $H(P) \leq \rho X_{k+1}$ from (14), for $\rho$ small enough .

Since the minimal polynomial $P_{\alpha_{k}}(X)$ satisfies (14), we deduce from Lemma 6.1 and from (13) the estimation

$$
X_{k}^{-1} X_{k+1}^{-2} \ll X_{k}\left|\xi_{\varphi}-\alpha_{k}^{\prime}\right|\left|\xi_{\varphi}-\alpha_{k}\right| \ll\left|P_{\alpha_{k}}\left(\xi_{\varphi}\right)\right| \ll X_{k+1}^{-w},
$$

which implies the upper bound

$$
w \leq 2+\frac{1}{\eta_{k}}+o(1)
$$

Let us recall that we have selected $k$ arbitrarily large with $w \geq 2+\frac{1}{\eta_{k}}+\epsilon$. The final contradiction follows. 


\section{Construction of simultaneous rational approximations to $\xi_{\varphi}, \xi_{\varphi}^{2}$.}

As for the Fibonacci continued fraction $\xi_{(\sqrt{5}-1) / 2}$ considered by Roy [22], the ordered sequence of palindromic prefixes of the mot $m_{\varphi}$ provides a sequence of simultaneous rational approximations to $\xi_{\varphi}, \xi_{\varphi}^{2}$. Let us recall the principle of the construction.

If the prefix $m_{\varphi \mid N}$ of the first $N$ letters of the word $m_{\varphi}$ is a palindrome, then the matrix

$$
M_{\mid N}=\left(\begin{array}{ll}
x_{0} & x_{1} \\
x_{1} & x_{2}
\end{array}\right)
$$

associated to the word $m_{\varphi \mid N}$ is symmetrical, and we have

$$
x_{0}=q_{N}, \quad x_{1}=p_{N}=q_{N-1}, \quad x_{2}=p_{N-1},
$$

where $p_{0} / q_{0}=0, p_{1} / q_{1}, \ldots$ denotes the sequence of convergents of $\xi_{\varphi}$. Thus,

$$
\frac{x_{2}}{x_{0}}=\frac{p_{N}}{q_{N}} \cdot \frac{p_{N-1}}{q_{N-1}}
$$

is a good rational approximation of $\xi_{\varphi}^{2}$. Setting

$$
\mathbf{x}=\left(x_{0}, x_{1}, x_{2}\right), \quad H(\mathbf{x})=\max \left\{x_{0}, x_{1}, x_{2}\right\} \quad \text { and } \quad L(\mathbf{x})=\max \left\{\left|x_{0} \xi_{\varphi}-x_{1}\right|,\left|x_{0} \xi_{\varphi}^{2}-x_{2}\right|\right\},
$$

it follows that

$$
L(\mathbf{x}) \gg \ll H(\mathbf{x})^{-1} .
$$

With some abuse of notation, we shall identify without ambiguity the integer triple $\mathbf{x}=\left(x_{0}, x_{1}, x_{2}\right)$ with the symmetrical matrix $M_{\mid N}$. Thus, we have

$$
\operatorname{det}(\mathbf{x})=x_{0} x_{2}-x_{1}^{2}= \pm 1
$$

since the matrix $\mathbf{x}$ is defined by a product of matrices of determinant -1 . It is also convenient to view $\mathbf{x}$ as the projective coordinates of a point in $\mathbf{P}^{2}$, and to investigate alignments of points in $\mathbf{P}^{2}$. Three points $\mathbf{x}, \mathbf{y}, \mathbf{z}$ are located on the same line in $\mathbf{P}^{2}$ if $\operatorname{det}(\mathbf{x}, \mathbf{y}, \mathbf{z})=0$.

Let us uniquely write each integer $\ell \geq 1$ in the form

$$
\ell=\ell_{k}+t \quad \text { where } \quad \ell_{k}=s_{2}+\cdots+s_{k}, \quad\left(\ell_{1}=0\right)
$$

with $k \geq 1$ and $1 \leq t \leq s_{k+1}$. We denote by $\mathbf{x}_{\ell}$ the triple associated to the palindrome $\left(m_{k}^{t} m_{k-1}\right)^{\prime}$ of index $(k, t)$ considered in Lemma 5.3. The following result describes the configuration in $\mathbf{P}^{2}$ of the sequence of points $\mathbf{x}_{\ell}$. 
Lemma 7.1. Let $k$ be an integer $\geq 3$.

(i) The point $\mathbf{x}_{\ell_{k-1}}$ and the $s_{k+1}+1$ consecutive points

$$
\mathbf{x}_{\ell_{k}+1}, \ldots, \mathbf{x}_{\ell_{k+1}+1}
$$

are located on the same projective line.

(ii) We have the formula

$$
\operatorname{det}\left(\mathbf{x}_{\ell_{k}}, \mathbf{x}_{\ell_{k}+1}, \mathbf{x}_{\ell_{k}+2}\right)= \pm(b-a) .
$$

Therefore, the three consecutive points $\mathbf{x}_{\ell_{k}}, \mathbf{x}_{\ell_{k}+1}, \mathbf{x}_{\ell_{k}+2}$ are linearly independent.

Proof. Notice that the point $\mathbf{x}_{\ell_{k-1}}$ is associated to the palindrome $m_{k-1}^{\prime}$. When $k \geq 3$ and $\ell=\ell_{k}+t$, with $1 \leq t \leq s_{k+1}$, the $\ell$-th palindrome considered in Lemma 5.3 is equal to $m_{k}^{t} m_{k-1}^{\prime}$. In terms of matrices $M_{j}$ and $M_{j}^{\prime}$, we have

$$
\mathbf{x}_{\ell_{k-1}}=M_{k-1}^{\prime}, \quad \mathbf{x}_{\ell_{k}+t}=M_{k}^{t} M_{k-1}^{\prime}
$$

for $t=1, \ldots, s_{k+1}$, and

$$
\mathbf{x}_{\ell_{k+1}+1}=M_{k+1} M_{k}^{\prime}=M_{k}^{s_{k+1}} M_{k-1} M_{k}^{\prime}=M_{k}^{1+s_{k+1}} M_{k-1}^{\prime},
$$

the last equality coming from the relation $m_{k-1} m_{k}^{\prime}=m_{k} m_{k-1}^{\prime}$ (cf. Lemma 5.1) at the level of the associated words. We observe that all these matrices are of the form $M_{k}^{t} M_{k-1}^{\prime}$ with $t=0,1, \ldots, s_{k+1}+1$. Then, the Cayley-Hamilton Theorem gives the linear relations

$$
\mathbf{x}_{\ell_{k}+2}=\operatorname{trace}\left(M_{k}\right) \mathbf{x}_{\ell_{k}+1} \pm \mathbf{x}_{\ell_{k-1}} \quad \text { and } \quad \mathbf{x}_{\ell_{k}+t+2}=\operatorname{trace}\left(M_{k}\right) \mathbf{x}_{\ell_{k}+t+1} \pm \mathbf{x}_{\ell_{k}+t}
$$

linking three consecutive points of the list (i).

Concerning the assertion (ii), we deduce by linearity from (19) that

$$
\operatorname{det}\left(\mathbf{x}_{\ell_{k}}, \mathbf{x}_{\ell_{k}+1}, \mathbf{x}_{\ell_{k}+2}\right)= \pm \operatorname{det}\left(\mathbf{x}_{\ell_{k}}, \mathbf{x}_{\ell_{k}+2}, \mathbf{x}_{\ell_{k}+3}\right)=\cdots= \pm \operatorname{det}\left(\mathbf{x}_{\ell_{k}}, \mathbf{x}_{\ell_{k+1}}, \mathbf{x}_{\ell_{k+1}+1}\right) .
$$

Now, we have

$$
\mathbf{x}_{\ell_{k}}=M_{k}^{\prime}, \quad \mathbf{x}_{\ell_{k+1}}=M_{k+1}^{\prime} \quad \text { and } \quad \mathbf{x}_{\ell_{k+1}+1}=M_{k+1} M_{k}^{\prime}
$$

Following Roy, let us set $J=\left(\begin{array}{cc}0 & 1 \\ -1 & 0\end{array}\right)$ and use his formula (2.1) from [23]:

$$
\operatorname{det}(\mathbf{x}, \mathbf{y}, \mathbf{z})=\operatorname{trace}(J \mathbf{x} J \mathbf{z} J \mathbf{y}),
$$

which is valid for all triples $\mathbf{x}, \mathbf{y}, \mathbf{z}$, identified as before to symmetrical matrices. Noting that $J \mathbf{x} J= \pm \mathbf{x}^{-1}$ if $\operatorname{det}(\mathbf{x})= \pm 1$, we obtain

$$
\begin{aligned}
\operatorname{det}\left(\mathbf{x}_{\ell_{k}}, \mathbf{x}_{\ell_{k+1}}, \mathbf{x}_{\ell_{k+1}+1}\right) & = \pm \operatorname{trace}\left(J M_{k}^{\prime}\left(M_{k+1} M_{k}^{\prime}\right)^{-1} M_{k+1}^{\prime}\right) \\
& = \pm \operatorname{trace}\left(J F_{k+1}^{-1}\right)= \pm(b-a),
\end{aligned}
$$

which is non zero since $a$ and $b$ are distinct.

Now, we are able to find the values of the functions $\lambda_{2}$ and $\hat{\lambda}_{2}$ at the point $\xi_{\varphi}$. 
Proposition 7.1. We have

$$
\lambda_{2}\left(\xi_{\varphi}\right)=1 \quad \text { and } \quad \hat{\lambda}_{2}\left(\xi_{\varphi}\right)=\frac{1+\sigma}{2+\sigma}
$$

Proof. The first formula $\lambda_{2}\left(\xi_{\varphi}\right)=1$ follows immediately from (16), since the partial quotients of $\xi_{\varphi}$ are bounded.

The proof of the second assertion is more elaborate. For any $\ell \geq 1$, let us set $X_{\ell}^{\prime}=H\left(\mathbf{x}_{\ell}\right)$ and denote by $\eta_{\ell}^{\prime}$ the exponent defined by the relation $X_{\ell+1}^{\prime}=X_{l}^{\prime} \eta_{\ell}^{\prime}$.

We first prove that

$$
\liminf _{\ell \geq 1}\left(\frac{1}{\eta_{\ell}^{\prime}}\right)=\frac{1+\sigma}{2+\sigma}
$$

One deduce from (11) and from the relations (17) and (18), the estimation

$$
X_{k}^{t} X_{k-1} \ll X_{\ell_{k}+t}^{\prime} \ll\left(2 X_{k}\right)^{t} X_{k-1}
$$

which is valid for $t=1, \ldots, s_{k+1}+1$ and for any integer $k \geq 3$. It follows that

$$
\eta_{\ell_{k}+t}^{\prime}=\frac{t+1+\frac{1}{\eta_{k-1}}}{t+\frac{1}{\eta_{k-1}}}\left(1+\mathcal{O}\left(\frac{1}{\log X_{k}}\right)\right) \leq \frac{2+\frac{1}{\eta_{k-1}}}{1+\frac{1}{\eta_{k-1}}}(1+o(1))
$$

for $t=1, \ldots, s_{k+1}$ and $k$ tending to infinity. Then Lemma 4.1 implies that

$$
\liminf _{\ell}\left(\frac{1}{\eta_{\ell}^{\prime}}\right)=\liminf _{k}\left(\frac{1+\frac{1}{\eta_{k}}}{2+\frac{1}{\eta_{k}}}\right)=\frac{1+\sigma}{2+\sigma} .
$$

The upper bound

$$
L\left(\mathbf{x}_{\ell}\right) \ll X_{\ell}^{\prime-1}
$$

is a special case of (16). Selecting in the interval $X_{\ell}^{\prime} \leq X<X_{\ell+1}^{\prime}$ the point $\mathbf{x}_{\ell}$ as a simultaneous rational approximation of $\left(\xi_{\varphi}, \xi_{\varphi}^{2}\right)$, we obtain immediately the lower bound

$$
\hat{\lambda}_{2}\left(\xi_{\varphi}\right) \geq \liminf _{\ell \geq 1}\left(\frac{1}{\eta_{\ell}^{\prime}}\right)=\frac{1+\sigma}{2+\sigma}
$$

In order to prove the equality $\hat{\lambda}_{2}\left(\xi_{\varphi}\right)=(1+\sigma) /(2+\sigma)$, we argue now by contradiction. The idea rests on the observation that the inferior limit of the sequence $1 / \eta_{\ell}^{\prime}$ is reached for indices $\ell$ of the form $\ell_{k}+1$, as (20) shows. If on the contrary, $\hat{\lambda}_{2}\left(\xi_{\varphi}\right)$ should be $>(1+\sigma) /(2+\sigma)$, let $\lambda$ be a real number in the interval

$$
\frac{1+\sigma}{2+\sigma}=\liminf _{\kappa}\left(\frac{1}{\eta_{\ell_{\kappa}+1}^{\prime}}\right)<\lambda<\hat{\lambda}_{2}\left(\xi_{\varphi}\right)
$$


Thus, there exist infinitely many integers $k$ such that

$$
\lambda \geq \frac{1}{\eta_{\ell_{k}+1}^{\prime}} .
$$

Furthermore, for each sufficiently large $X$, there exists some non zero integer point $\mathbf{x}$ such that

$$
H(\mathbf{x}) \leq X \quad \text { and } \quad L(\mathbf{x}) \leq X^{-\lambda}
$$

Choose $X=\rho X_{\ell_{k}+2}^{\prime}$, for some positive constant $\rho$ independent of $k$, and observe that

$$
X_{\ell_{k}}^{\prime}=X_{k}, \quad X_{\ell_{k}+1}^{\prime} \gg \ll X_{k} X_{k-1}, \quad X_{\ell_{k}+2}^{\prime} \gg \ll X_{k}^{2} X_{k-1} .
$$

Then we deduce from (22) and from (23) that

$$
L(\mathbf{x}) \leq X^{-\lambda} \leq \rho^{-\lambda} X_{\ell_{k}+1}^{\prime-1} \ll \rho^{-\lambda}\left(X_{k} X_{k-1}\right)^{-1}
$$

Now we prove that

$$
\operatorname{det}\left(\mathbf{x}, \mathbf{x}_{\ell_{k}+1}, \mathbf{x}_{\ell_{k}}\right)=\operatorname{det}\left(\mathbf{x}, \mathbf{x}_{\ell_{k}+1}, \mathbf{x}_{\ell_{k}+2}\right)=0
$$

for $k$ sufficiently large, if the constant $\rho$ is small enough. Proceeding as in Lemma 4 of [13], and using the estimations (21),(23) and (24), we bound

$$
\begin{aligned}
\left|\operatorname{det}\left(\mathbf{x}, \mathbf{x}_{\ell_{k}+1}, \mathbf{x}_{\ell_{k}}\right)\right| & \ll X_{\ell_{k}+1}^{\prime} L\left(\mathbf{x}_{\ell_{k}}\right) L(\mathbf{x})+X_{\ell_{k}}^{\prime} L\left(\mathbf{x}_{\ell_{k}+1}\right) L(\mathbf{x})+X L\left(\mathbf{x}_{\ell_{k}}\right) L\left(\mathbf{x}_{\ell_{k}+1}\right) \\
& \ll \rho^{-\lambda} X_{k}^{-1}+\rho^{-\lambda} X_{k}^{-1} X_{k-1}^{-2}+\rho
\end{aligned}
$$

and

$$
\begin{aligned}
\left|\operatorname{det}\left(\mathbf{x}, \mathbf{x}_{\ell_{k}+1}, \mathbf{x}_{\ell_{k}+2}\right)\right| & \ll X_{\ell_{k}+2}^{\prime} L\left(\mathbf{x}_{\ell_{k}+1}\right) L(\mathbf{x})+X_{\ell_{k}+1}^{\prime} L\left(\mathbf{x}_{\ell_{k}+2}\right) L(\mathbf{x})+X L\left(\mathbf{x}_{\ell_{k}+2}\right) L\left(\mathbf{x}_{\ell_{k}+1}\right) \\
& \ll \rho^{-\lambda} X_{k-1}^{-1}+\rho^{-\lambda} X_{k}^{-2} X_{k-1}^{-1}+\rho\left(X_{k} X_{k-1}\right)^{-1} .
\end{aligned}
$$

These two determinants being integers, both necessarily vanish if $\rho$ is small and if the index $k$ is large enough.

The assertion (ii) of Lemma 7.1 then implies that $\mathbf{x}$ is an integer multiple of $\mathbf{x}_{\ell_{k}+1}$, since this last point has relatively prime coordinates. Thus

$$
X_{\ell_{k}+1}^{\prime-1} \ll L\left(\mathbf{x}_{\ell_{k}+1}\right) \leq L(\mathbf{x}) \leq\left(\rho X_{\ell_{k}+2}^{\prime}\right)^{-\lambda}=\rho^{-\lambda} X_{\ell_{k}+1}^{\prime}{ }^{-\lambda \eta_{\ell_{k}+1}^{\prime}} .
$$

We obtain

$$
\lambda \leq \frac{1+o(1)}{\eta_{\ell_{k}+1}^{\prime}},
$$

from which follows the upper bound $\lambda \leq \liminf _{\kappa}\left(1 / \eta_{\ell_{\kappa}+1}^{\prime}\right)$, since the above bound holds for infinitely many $k$. This last upper bound contradicts our choice of $\lambda$. [ 


\section{Questions of spectra.}

By abuse of language, we call spectrum of a function the set of values taken by this function at transcendental points. In view of Theorem 2.4, this definition is by no means restrictive when we consider the six exponents of approximation studied in the present article.

We start with a brief review of known results about the functions $w_{n}$ and $w_{n}^{*}$. Thank to exact computations of Hausdorff dimensions, Baker \& Schmidt [4] have shown that the spectrum of the function $w_{n}^{*}$ contains $[n,+\infty]$. We have equality if the Wirsing Conjecture, recalled in the introduction, holds. In a long and difficult article, Bernik [6] has established that the spectrum of the function $w_{n}$ is equal to the interval $[n,+\infty]$. As far as we know, the study of the spectrum of $\lambda_{n}$ remains to be performed. Nevertheless, it is easily seen that this spectrum is unbounded. Remark also that the functions $w_{n}$ and $w_{n}^{*}$ differ at some transcendental points $[5,9]$.

Unlike $w_{n}, w_{n}^{*}$ and $\lambda_{n}$, the functions $\hat{w}_{n}, \hat{w}_{n}^{*}$ and $\hat{\lambda}_{n}$ are bounded and their spectrum is, respectively, contained in the intervals $[n, 2 n-1],[1,2 n-1]$ and $\left[1 / n,(\lceil n / 2\rceil)^{-1}\right]$, for any degree $n \geq 1$.

Now, let us look more carefully at the spectra of the functions $\hat{w}_{2}$ and $\hat{\lambda}_{2}$, respectively contained in the more restrained intervals $[2,(3+\sqrt{5}) / 2]$ and $[1 / 2,(\sqrt{5}-1) / 2]$, according to Theorem 2.6. Our Theorem 3.1 points out a lot of values in each of these quadratic spectra, and it invites us to study the set $\mathcal{S}$ of values taken by the quantity

$$
\sigma=\frac{1}{\limsup _{k \geq 1}\left[s_{k} ; s_{k-1}, \ldots, s_{1}\right]},
$$

for any bounded sequence $s_{k}$ of positive integers. It is easily seen that the largest element in $\mathcal{S}$ is $(\sqrt{5}-1) / 2 \simeq 0.618 \ldots$ which is obtained when 1 is the only integer appearing infinitely many times in the sequence $s_{k}$. The values immediately below have been found by Cassaigne [11]. They constitute a decreasing sequence of quadratic numbers converging to the largest accumulation point of $\mathcal{S}$. His result, formulated in Theorem 2 of [11], is the following:

Theorem 8.1. Denote by $\psi$ the endomorphism of the monoïd $\{1,2\}^{*}$ defined by

$$
\psi(1)=2 \quad \text { and } \quad \psi(2)=211
$$

The decreasing sequence of quadratic numbers

$$
\sigma_{n}=\left[0 ; \psi^{n}(1), \psi^{n}(1), \ldots\right] \quad(n \geq 0)
$$

converges to the real number

$$
s=\left[0 ; \lim _{n \rightarrow \infty} \psi^{n}(1)\right]=[0 ; 2,1,1,2,2,2,1,1, \ldots] \simeq 0.38674997056 \ldots
$$


The point $s$ is the largest accumulation point of $\mathcal{S}$ and the intersection of $\mathcal{S}$ with the interval $] s,+\infty$ ] coincides with the set of values taken by the sequence $\left(\sigma_{n}\right)_{n \geq 0}$.

In Theorem 8.1, the sequence of iterated words $\psi^{n}(1)$ converges to the infinite word $21122211 \ldots$, the fixed point of the substitution $\psi$ acting on $\{1,2\}^{\mathbf{N}}$. Remark also that $\sigma_{n}=\sigma_{\varphi}$ for the choice of angle $\varphi=\left[0 ; \widetilde{\psi^{n}(1)}, \widetilde{\psi^{n}(1)}, \ldots\right]$, where the symbol $\sim$ stands for the mirror of a word.

Thus the three largest values of $\hat{w}_{2}(\xi)$, when $\xi$ is any Sturmian continued fraction, are

$$
\frac{3+\sqrt{5}}{2}>1+\sqrt{2}>\frac{4+\sqrt{10}}{3}>\cdots
$$

while those of $\hat{\lambda}_{2}(\xi)$ are

$$
\frac{\sqrt{5}-1}{2}>2-\sqrt{2}>\frac{-2+\sqrt{10}}{2}>\cdots
$$

Then, a natural question arises: where are located these values inside the larger spectra of the values $\hat{w}_{2}(\xi)$ and $\hat{\lambda}_{2}(\xi)$, when $\xi$ now ranges among all transcendental real numbers ? If we exclude extremal numbers of Roy for which we simultaneously have $\hat{w}_{2}(\xi)=(3+\sqrt{5}) / 2$ and $\hat{\lambda}_{2}(\xi)=(\sqrt{5}-1) / 2$, does there exist real numbers $\xi$ such that $\hat{w}_{2}(\xi)>1+\sqrt{2}$, or $\hat{\lambda}_{2}(\xi)>2-\sqrt{2}$ ? In this respect, one can remark that other combinatorial constructions lead to real numbers $\xi$ for which $\hat{w}_{2}^{*}(\xi)>2$ and $\hat{\lambda}_{2}(\xi)>1 / 2$. As an example, we can consider the number $\xi$ whose partial quotients are given by the fixed point of the so-called Tribonacci substitution defined by $1 \rightarrow 12,2 \rightarrow 13$ and $3 \rightarrow 1$.

Besides, Cassaigne has shown in [11] that the subset $\mathcal{S} \subset \mathbf{R}$ has an empty interior. It should be interesting to know whether the same result holds for the spectra of the functions $\hat{w}_{2}$ and $\hat{\lambda}_{2}$.

Let us finally point out that the study of the set $\mathcal{S}$ below its greatest accumulation point $s$ remains to be done.

\section{References}

[1] W. W. Adams and J. L. Davison, A remarkable class of continued fractions, Proc. Amer. Math. Soc. 65 (1977), 194-198.

[2] J.-P. Allouche, J. L. Davison, M. Queffélec, and L. Q. Zamboni, Transcendence of Sturmian or morphic continued fractions, J. Number Theory 91 (2001), 39-66.

[3] B. Arbour and D. Roy, A Gel'fond type criterion in degree two, Acta Arith. 11 (2004), 97-103. 
[4] A. Baker and W. M. Schmidt, Diophantine approximation and Hausdorff dimension, Proc. London Math. Soc. 21 (1970), 1-11.

[5] R. C. Baker, On approximation with algebraic numbers of bounded degree, Mathematika 23 (1976), 18-31.

[6] V. I. Bernik, Application of the Hausdorff dimension in the theory of Diophantine approximations, Acta Arith. 42 (1983), 219-253 (in Russian). English translation in Amer. Math. Soc. Transl. 140 (1988), 15-44.

[7] Y. Bugeaud, On the approximation by algebraic numbers with bounded degree, Algebraic number theory and Diophantine analysis (Graz, 1998), 47-53, de Gruyter, Berlin, 2000.

[8] Y. Bugeaud, Approximation par des nombres algébriques, J. Number Theory 84 (2000), $15-33$.

[9] Y. Bugeaud, Mahler's classification of numbers compared with Koksma's, Acta Arith. 110 (2003), 89-105.

[10] Y. Bugeaud, Approximation by algebraic numbers, Cambridge Tracts in Mathematics, Cambridge University Press. To appear.

[11] J. Cassaigne, Limit values of the recurrence quotient of Sturmian sequences, Theor. Comput. Sci. 218 (1999), 3-12.

[12] H. Davenport and W. M. Schmidt, Approximation to real numbers by quadratic irrationals, Acta Arith. 13 (1967), 169-176.

[13] H. Davenport and W. M. Schmidt, Approximation to real numbers by algebraic integers, Acta Arith. 15 (1969), 393-416.

[14] H. Davenport and W. M. Schmidt, Dirichlet's theorem on Diophantine approximation, Symposia Mathematica, Vol. IV (INDAM, Rome, 1968/69), pp. 113-132, Academic Press, London, 1970.

[15] J. L. Davison, A series and its associated continued fraction, Proc. Amer. Math. Soc. 63 (1977), 29-32.

[16] J. F. Koksma, Über die Mahlersche Klasseneinteilung der transzendenten Zahlen und die Approximation komplexer Zahlen durch algebraische Zahlen, Monats. Math. Phys. 48 (1939), 176-189.

[17] M. Laurent, Some remarks on the approximation of complex numbers by algebraic numbers. Proceedings of the 2nd Panhellenic Conference in Algebra and Number Theory (Thessaloniki, 1998). Bull. Greek Math. Soc. 42 (1999), 49-57.

[18] M. Laurent, Simultaneous rational approximation to the successive powers of a real number. Indag. Math. 11 (2003), 45-53.

[19] K. Mahler, Zur Approximation der Exponentialfunktionen und des Logarithmus. I, II, J. reine angew. Math. 166 (1932), 118-150. 
[20] M. Queffélec, Approximations diophantiennes des nombres sturmiens, J. Théor. Nombres Bordeaux 14 (2002), 613-628.

[21] A. M. Rockett and P. Szüsz, Continued Fractions, World Scientific, Singapore, 1992.

[22] D. Roy, Approximation simultanée d'un nombre et son carré, C. R. Acad. Sci. Paris 336 (2003), 1-6.

[23] D. Roy, Approximation to real numbers by cubic algebraic numbers, I, Proc. London Math. Soc. 88 (2004), 42-62.

[24] D. Roy, Approximation to real numbers by cubic algebraic numbers, II, Ann. of Math. 158 (2003), 1081-1087.

[25] D. Roy, Diophantine approximation in small degree, Preprint.

[26] V. G. Sprindžuk, Mahler's problem in metric number theory, Amer. Math. Soc., Transl. math. monogr. 25, Providence, R. I., 1969.

[27] E. Wirsing, Approximation mit algebraischen Zahlen beschränkten Grades, J. reine angew. Math. 206 (1961), 67-77.

Yann Bugeaud

Université Louis Pasteur

U. F. R. de mathématiques

7 , rue René Descartes

67084 STRASBOURG (FRANCE)

bugeaud@math.u-strasbg.fr
Michel Laurent

Institut de Mathématiques de Luminy C.N.R.S. - U.P.R. 9016 - case 907

163, avenue de Luminy 13288 MARSEILLE CEDEX 9 (FRANCE) laurent@iml.univ-mrs.fr 